\title{
Volumentherapie in der Sepsis: Wann ist das Limit erreicht?
}

\author{
Elke Muhl \\ Chirurgische Intensivstation, Klinik für Chirurgie, Universitätsklinikum Schleswig-Holstein, Campus Lübeck, Deutschland
}

Schlüsselwörter

Sepsis · Volumentherapie - Vorlast · Monitoring · Kolloide

\section{Zusammenfassung}

Hintergrund: Die Endpunkte der Volumentherapie bei schwerer Sepsis und septischem Schock sind nicht selten Gegenstand kontroverser Diskussionen am Krankenbett. Methode: Anhand der Leitlinien und der aktuellen Literatur werden die Zielparameter sowie die durch Volumentherapie erreichbaren Wirkungen und Nebenwirkungen vorgestellt. Ergebnisse: Werden die Zielparameter der Volumentherapie in der Sepsis nicht in den ersten $6 \mathrm{~h}$ erreicht, ist ein erweitertes hämodynamisches Monitoring zur Überwachung der Vorlast und der Volumenreagibilität sowie der Gewebsoxygenierung indiziert. Eine Überinfusion führt nicht selten zu grotesken Anasarka und Nebenwirkungen am Herz, an der Lunge und im Abdomen, die lebensbedrohliche Ausmaße annehmen können. Schlussfolgerung: Die Grenzen der Volumentherapie sind nicht nur in hämodynamischen Zielparametern zu sehen, sondern auch in den Nebenwirkungen der Volumentherapie, die im Rahmen der Sepsisbehandlung nicht sämtlich vermieden, aber in ihren Auswirkungen begrenzt werden können.

\section{Einleitung}

Ein zentraler Pfeiler der supportiven Therapie in der Sepsis ist die Volumentherapie. Die Bedeutung einer frühzeitigen und raschen Volumentherapie in der Sepsis ist durch die eindrucksvollen Ergebnisse der Studie von Rivers et al. [1] gut belegt. In der schweren Sepsis und im septischen Schock sind
Keywords

Sepsis - Volume resuscitation · Preload - Monitoring · Colloids

\section{Summary}

Volume Resuscitation in Severe Sepsis: What Is the Limit?

Background: The endpoints of volume resuscitation in severe sepsis and septic shock are still a subject of discussion at the bedside. Method: Based on guidelines and current literature, recommended end points as well as the results and side effects of volume resuscitation are reported on. Results: If volume resuscitation is not successful within $6 \mathrm{~h}$, additional hemodynamic monitoring is recommended for measuring surrogate parameters of tissue oxygenation and preload as well as for predicting volume responsiveness. Over-resuscitation may result in anasarca and life-threatening side effects such as fluid lung, abdominal compartment syndrome, and decrease in cardiac output. Conclusion: Adverse effects of volume resuscitation and over-resuscitation are not always avoidable; however, adequate monitoring may limit negative effects on the patients' outcome.

\section{KARGER \\ Fax +497614520714 \\ Information@Karger.com}

www.karger.com (c) 2013 S. Karger GmbH, Freiburg

$1662-6664 / 13 / 0291-0034 \$ 38.00 / 0$

Accessible online at:

www.karger.com/vim nicht selten positive Flüssigkeitsbilanzen großen Ausmaßes erforderlich, um die Patienten hämodynamisch zu stabilisieren. Menge, Zielgrößen und Art des Volumenersatzes waren und sind - insbesondere während der letzten Jahre - Gegenstand wissenschaftlicher Arbeiten.

In der elektiven Viszeralchirurgie wurden negative Auswirkungen umfänglicher Volumensubstitution für die Heilung 
von gastrointestinalen Anastomosen und die Inzidenz von postoperativen Komplikationen belegt [2-4]. Somit stellt sich gerade bei der abdominellen Sepsis auch die Frage: «Wie viel Volumen ist genug?» Oder anders ausgedrückt: Sind Anasarka und «Fluid Lung» nach Therapie des septischen Schocks bei kapillärem Leck-Syndrom ein lässlicher Schönheitsfehler oder relevant für Morbidität und Mortalität der Patienten?

\section{Leitlinienempfehlungen zur Volumentherapie in der Sepsis}

In den Leitlinien der Sepsisgesellschaft [5] wird die Volumentherapie als erste Maßnahme der hämodynamischen Stabilisierung empfohlen (Empfehlungsgrad A, Evidenzgrad Ic). Initial sollen $20-40 \mathrm{ml} / \mathrm{kg}$ Körpergewicht $(\mathrm{KG})$ so rasch wie möglich infundiert werden, wenn eine Hypotonie oder eine Serum-Laktat-Erhöhung vorliegen. Dabei werden als Zielparameter der Volumentherapie in der Frühphase der Sepsis angegeben (Empfehlungsgrad C, Evidenzgrad IIc für $\mathrm{ScVO}_{2}$ ):

- Zentralvenöser Druck $\geq 8$ bzw. $\geq 12$ mm Hg unter mechanischer Beatmung

- Mittlerer arterieller Druck $\geq 65 \mathrm{~mm} \mathrm{Hg}$

- Diurese $\geq 0,5 \mathrm{ml} / \mathrm{kg} \mathrm{KG} / \mathrm{h}$

- $\mathrm{ScVO}_{2} \geq 70 \%$

- Serum-Laktat $\leq 1,5 \mathrm{mmol} / \mathrm{l}$ bzw. Abfall desselben.

Das rasche Erreichen dieser Zielkriterien hat eine geringere Mortalität der Sepsis zur Folge, wobei nicht ausschließlich die Volumentherapie, sondern ein ganzes Bündel von Maßnahmen in den zugrunde liegenden Studien zum Erfolg führte, unter anderem auch die frühzeitige Antibiotikatherapie. Die Bewertung des Erfolgs der Volumentherapie allein in der Sepsistherapie ist derzeit also kaum möglich [6].

Wenn sich die Therapieziele unter Volumenzufuhr nicht rasch erreichen lassen, wird der Einsatz von Vasopressoren (Norepinephrin) zur Erreichung eines adäquaten arteriellen Mitteldrucks (>65 mm Hg) (Empfehlungsgrad B, Evidenzgrad Ic) empfohlen. Positiv inotrope Substanzen kommen dann zum Einsatz, wenn trotz Volumentherapie ein eingeschränktes Herzzeitvolumen besteht. Als Medikament der ersten Wahl empfehlen die Leitlinien Dobutamin; gegebenenfalls kann auch Adrenalin zum Einsatz kommen [7].

In der Leitlinie [5] wird der Einsatz von Hydroxyethylstärke (HES) (HES-Lösungen 200/0,5 und 200/0,62) für die Volumensubstitution bei Patienten mit schwerer Sepsis und septischem Schock ausdrücklich nicht empfohlen (Empfehlung Grad A, Evidenzgrad Ia). Diese Empfehlung basiert auf den Ergebnissen der VISEP-Studie [7], in der Sepsis-Patienten unter Volumentherapie mit HES 200/0,5 im Vergleich zu Patienten, die eine modifizierte Ringer-Laktat-Lösung bekamen, eine um $12 \%$ erhöhte Inzidenz von akutem Nierenversagen und eine Verdoppelung der Notwendigkeit für den Einsatz einer Nierenersatztherapie hatten. Die negativen Effekte von HES auf die Nierenfunktion waren dosisabhängig. Sie traten aber auch dann auf, wenn eine Tagesdosis von $22 \mathrm{ml} / \mathrm{kg}$ $\mathrm{KG}$ nie überschritten wurde. Bei Patienten, die eine hohe kumulative Dosis HES erhalten hatten (136 ml/kg KG), war die 90-Tage-Sterblichkeit um $17 \%$ erhöht.

Seit Juli 2012 liegt eine kontrollierte randomisierte verblindete Vergleichsstudie vor, die die Volumentherapie bei schwerer Sepsis mit einer «modernen» niedrigmolekularen HES-Lösung $(130 / 0,4)$ gegenüber einer Ringer-AcetatLösung untersucht hat [8]. In der Studie wurde eine Dosisbegrenzung von $33 \mathrm{ml} / \mathrm{kg} \mathrm{KG}$ (Idealgewicht) eingehalten. Bei höherem Volumenbedarf wurde mit unverblindeter RingerAcetat-Lösung weiterbehandelt. Nur 2 Patienten in der Studienpopulation bekamen HES in einer Dosis, die die Maximaldosisempfehlung des Herstellers überschritt. Für die Endpunkte Mortalität und Notwenigkeit einer Nierenersatztherapie nach 90 Tagen war das Risiko in der HES-Gruppe erhöht: Mortalität 51 vs. $43 \%$ (relatives Risiko 1,17; 95\%-Konfidenzintervall 1,01-1,36; $\mathrm{p}=0,03$ ); Nierenersatztherapie 22 vs. $16 \%$ (relatives Risiko 1,35; Konfidenzintervall 1,01-1,80; $\mathrm{p}=0,04$ ). Die Empfehlung, in der schweren Sepsis keine HES-Lösungen zur Volumensubstitution zu verwenden, gilt somit auch für niedermolekulare HES-Lösungen.

Bezüglich der Endpunkte der Volumensubstitution, des hämodynamischen Monitorings sowie anderer kreislaufstabilisierender Maßnahmen und weiterer Therapiemaßnahmen in der Sepsis war die Studie sehr liberal angelegt. Die teilnehmenden Zentren sollten lediglich bestätigen, dass sie internationalen Leitlinien zur Sepsistherapie folgen. Somit sind Schlussfolgerungen zu den Endpunkten der Volumentherapie aus den genannten Studien nicht herzuleiten.

Seit 2011 ist in die Leitlinie aufgenommen, dass der Einsatz von Humanalbumin 5\% immerhin zu erwägen sei (im Gegensatz zur früheren ausdrücklichen Nichtempfehlung von Humanalbumin), wenn auch nur mit dem Evidenzgrad V (Expertenmeinung oder basierend auf Laborforschung/physiologischen Modellen). Diese Aussage beruht auf den Ergebnissen der SAFE-Study-Investigators, die in einer randomisierten Vergleichsstudie bei Patienten mit schwerer Sepsis keinen Unterschied in den Gruppen zwischen Albumin und physiologischer Kochsalzlösung bezüglich des Effekts auf Nierenfunktion und andere Organversagen [9] sowie einen Trend zu einem Überlebensvorteil fanden. Humanalbumin ist teuer und bietet als Lösung zur Volumensubstitution bezüglich der Mortalität - über alle Studien betrachtet - keine Vorteile gegenüber physiologischer Kochsalzlösung oder RingerLaktat-/Ringer-Acetat-Lösungen [10]. Humanalbumin-Lösungen als Volumenersatz haben somit vorerst nur in gut durchdachten randomisierten kontrollierten Studien ihren Platz.

Gelatinelösungen als kolloidaler Volumenersatz werden in der Sepsisleitlinie nicht erwähnt. Sie beeinträchtigen die Blutgerinnung durch Reduktion des Spiegels des von-WillebrandFaktors und von Faktor VIII, kompromittieren die Thrombo- 
Tab. 1. Volumentherapie in der Sepsis zwischen «Skylla und Charybdis»

\begin{tabular}{ll}
\hline Überinfusion & Unterinfusion, septischer Schock \\
\hline Ödeme peripher & $\begin{array}{l}\text { hohe Blutviskosität ( } \rightarrow \text { Verminderung der } \\
\text { Gewebsoxygenierung, Thrombosen) } \\
\text { unzureichender Perfusionsdruck }\end{array}$ \\
$\begin{array}{l}\text { Ödeme im Abdomen } \\
\text { Darmatonie }\end{array}$ & $\begin{array}{l}\text { Nierenversagen } \\
\text { Darmwandödem mit Perfusionsstörung }\end{array}$ \\
Aszites & Minderperfusion des Darms \\
Erhöhter intraabdomineller Druck / & \\
$\quad$ abdominelles Kompartmentsyndrom & \\
Verminderter Rückfluss zum rechten Herzen / & \\
$\quad$ Cava-Kompression & \\
Erhöhte Beatmungsdrucke & \\
Darmischämie & \\
Nierenversagen & \\
Kardiale Belastung / Pumpversagen & \\
Lungenstauung/-ödem, «Fluid Lung» & \\
Pleuraergüsse & \\
Respiratorische Insuffizienz & Myokardischämie \\
Leberstauung & \\
&
\end{tabular}

zytenfunktion und weisen ein hohes Risiko für anaphylaktische Reaktionen auf [11].

Zur hämodynamischen Stabilisierung wird somit primär die Anwendung von kristallinen Lösungen empfohlen (Empfehlungsgrad B, Evidenzgrad Ib) [5]. Bei Verwendung kristalliner Lösungen mit hohem Natrium- und Chloridgehalt ist zu bedenken, dass es zu einer hyperchlorämischen Azidose kommen kann. Auch bei kolloidalen Lösungen kann der Chloridgehalt hoch sein.

\section{Negative Effekte von massiver Volumensubstitution und Volumenüberladung}

Beim Nierengesunden steigt unter übermäßiger Volumenzufuhr die Diurese. Eine Hypervolämie führt via Freisetzung von ANP (Atriales Natriuretisches Peptid) zu einer vermehrten Ausscheidung von Natrium und Wasser [12], sodass ein Überwässerungsproblem gar nicht auftritt. Bei der relativen Hypovolämie in der Sepsis und bei Leberzirrhose kommt es jedoch zu Flüssigkeitsverschiebungen. Sowohl Über- als auch Unterinfusionen können negative Effekte haben (Tab. 1).

Bei Patienten nach septischem Schock mit kapillärem Leck-Syndrom und massiver Volumensubstitution funktioniert dieser Mechanismus daher nur bedingt, insbesondere wenn eine Niereninsuffizienz hinzukommt und vielleicht sogar zusätzlich eine Herzinsuffizienz und/oder koronare Herzerkrankung die Verträglichkeit einer Volumenbelastung vermindern. Periphere Ödeme, «Fluid Lung» und Pleuraergüsse, Ödem intraabdominell, Leberstauung, Aszites, bei Darmödem und venöser Stauung des Darms auch Darmatonie/-paralyse, abdominelles Kompartmentsyndrom, abnehmendes Herzzeitvolumen unter Volumenzufuhr, Unter- kühlung, Hypoalbuminäme, hyperchlorämische Azidose, Verdünnungskoagulopathie und Verdünnungsanämie sind dann als direkte Folgen von (relativer) Volumenüberladung zu sehen.

Der Anämie kann mit Gabe von Erythrozytenkonzentraten und Anhebung des Hämatokrit auf 30\% (Zielwert in den Sepsisleitlinien) leicht begegnet werden [6].

Bei hohen Temperaturen in der Sepsis ist zur Verminderung des Sauerstoffverbrauchs die Kühlung durch Infusionslösungen erwünscht. Eine Unterkühlung muss allerdings vermieden werden, da sie zu einer Vasokonstriktion und Minderperfusion peripher und somit gegebenenfalls zur Verstärkung einer Azidose sowie zu einer verminderten Wirksamkeit der Katecholamine führt. Auch beim septischen Patienten muss somit bei massivem Volumenbedarf frühzeitig an die Wärmung der Infusionslösungen und/oder des Patienten gedacht werden.

Die Verdünnungskoagulopathie allein ist meist nicht die Ursache von Blutungen. Sie kann allerdings bei großen operativen Eingriffen klinisch relevant werden, besonders dann, wenn sie additiv zu einer durch die Sepsis hervorgerufenen Koagulopathie (disseminierte intravasale Koagulopathie, DIC) wirkt. Ab einer Hämodilution von $60 \%$ und mehr wird die Koagulabilität beeinträchtigt [11].

Eine hyperchlorämische Azidose kann durch kristallinen Volumenersatz in Form von Ringer-Lösung oder NaClLösung (in geringerem Maße durch balancierte RingerLösung/Ringer-Laktat/-Acetat, die eine geringere Chloridkonzentration enthalten) entstehen. Eine hyperchlorämische Azidose führt zu einer Vasokonstriktion am Vas afferens in der Niere und zu einer Verminderung der viszeralen Perfusion. Ob diesem Faktum eine klinische Relevanz zukommt, ist unklar. 
Die Expansion des Extrazellulärraums durch aggressive Volumensubstitution in der Sepsis führt nicht nur zur Verdünnung von Gerinnungsfaktoren, sondern auch zu verminderten Serum- und Gewebsspiegeln von Medikamenten, die sich im Körperwasser verteilen. Hierzu gehört auch eine Reihe von Antibiotika.

Die Hypalbuminämie, ein Verdünnungseffekt bei massiver kristalliner Volumensubstitution, führt über die Herabsetzung des onkotischen Drucks zu interstitiellen Ödemen. Die Bedeutung der Hypalbuminämie in der Sepsis liegt darüber hinaus in ihrem Einfluss auf die Albuminbindung vieler Medikamente. Kapilläres Leck-Syndrom und erniedrigtes SerumAlbumin addieren sich in ihrer pharmakokinetischen Wirkung. Sie führen zu einem erhöhten Verteilungsvolumen und verminderten Spitzenspiegeln bei Medikamenten mit mittlerer bis hoher Eiweißbindung und geringem bis mittlerem Verteilungsvolumen, sodass eine erhöhte Loading-Dose benötigt wird. Dies gilt z.B. auch für $\beta$-Laktam-Antibiotika und Glykopeptide [13].

Periphere Ödeme stellen für sich genommen zunächst kein Problem dar, auch wenn sie optisch sehr eindrucksvoll sein können. Allerdings haben die Patienten ein erhöhtes Dekubitusrisiko, dem mit vorbeugenden Maßnahmen Rechnung getragen werden muss. Pleuraergüsse sind durch Punktion und Drainage einer Therapie zugänglich.

Aszites, Darmwandödem und venöse Stauung des Darms nach massiver Volumensubstitution können zu erhöhtem abdominellem Druck und zu Darmatonie/-paralyse bis hin zum abdominellen Kompartmentsyndrom führen. Die damit einhergehende Durchblutungsverminderung des Darms kann unter anderem Störungen der Anastomosenheilung beim viszeralchirurgischen Patienten und Nierenversagen bewirken. Therapiebedürftig wird der erhöhte abdominelle Druck dann, wenn:

1. eine zunehmende Erhöhung der Beatmungsdrucke erforderlich wird,

2. das Abdomen fest und nicht eindrückbar ist,

3. der intraabdominelle Druck $>20 \mathrm{~mm} \mathrm{Hg}$ oder

4. der abdominelle Perfusiondruck (APP $=$ mittlerer arterieller Druck; IAP = intraabdomineller Druck) $<60 \mathrm{~mm} \mathrm{Hg}$ beträgt und z.B. die Nierenperfusion und damit auch die Diurese abnimmt,

5. ein Anstieg von Serum-Laktat und Azidose auftreten (beim Trauma-Patienten auch Hirndruckanstieg).

Die Empfehlungen der World Society of the Abdominal Compartment Syndrome [14] raten zur chirurgischen Dekompression des Abdomens (Grad 1c), wenn konservative Therapiemaßnahmen versagen, was bei Patienten mit septischem Schock und nach massiver Volumengabe in aller Regel der Fall sein wird. Als konservative Maßnahmen stehen zur Verfügung: abführende Maßnahmen/Prokinetika, Absaugen der Magensonde, Sedierung, Entwässerung durch Diuretika/ Nierenersatztherapie, Entfernung einengender Verbände und ein ausreichender arterieller Blutdruck (APP >60 mm Hg).
Das Herzzeitvolumen ist im Volumenmangel oft erhöht und sollte sich - wenn keine anderen kardialen Erkrankungen dies behindern - unter Volumenzufuhr normalisieren. Eine Erhöhung der Vorlast darüber hinaus wird dann wieder zu einer Verminderung des Schlagvolumens und des Herzzeitvolumens führen (Frank-Starling-Mechanismus).

Eine Herausforderung ist der septische Patient mit hohem Volumenbedarf und vorbestehender Herzinsuffizienz. Bei diesen Patienten ist der frühzeitige Einsatz von Inotropika (Dobutamin) und Vasopressoren angezeigt und eine kontinuierliche Messung von Herzzeitvolumen und Vorlast-Parametern unabdingbar. Klassische Therapeutika der akuten Herzinsuffizienz wie z.B. Phosphodiesterasehemmer verbieten sich in der Sepsis in aller Regel, da sie den ohnehin erniedrigten peripheren Gefäßwiderstand weiter senken und zu nicht erwünschten Blutdruckabfällen führen.

Eine wirkliche Grenze der Volumenbelastbarkeit eines septischen Patienten ist die «Fluid Lung» mit Lungenversagen. Ist der Patient schon mit hohem PEEP (positiver endexpiratorischer Druck) und hohem $\mathrm{FiO}_{2}$ beatmet und kommt es zu einer Verschlechterung der Oxygenierung unter Flüssigkeitszufuhr, ist eine Grenze erreicht. In diesen Fällen können Nierenersatzverfahren unabhängig vom Nierenversagen zur Elimination eines Zuviels an Volumen eingesetzt werden.

\section{Monitoring}

Die Zielparameter der Volumentherapie der Sepsis sind oben genannt. Zum Basismonitoring gehört demnach mindestens die Erfassung dieser Parameter: arterieller Blutdruck, Herzfrequenz, Diurese, zentralvenöse Sauerstoffsättigung, SerumLaktat. Zur «Grundausstattung» eines Patienten mit schwerer Sepsis und septischem Schock gehören also ein zentraler Venenzugang, eine arterielle Druckmessung, ein Blasenkatheter und großvolumige venöse Zugänge für die Volumenzufuhr. Zentralvenöse Sauerstoffsättigung und Serum-Laktat können diskontinuierlich gemessen werden.

Die kontinuierliche Messung der zentralvenösen Sauerstoffsättigung über einen entsprechend ausgestatteten zentralen Venenkatheter und die kontinuierliche Temperaturüberwachung durch einen entsprechend ausgestatteten Blasenkatheter erleichtern die Überwachung dieser Parameter und die schnelle therapeutische Reaktion.

Die kontinuierliche Messung wird insbesondere dann sinnvoll sein, wenn der Patient nicht innerhalb der ersten Stunde durch Volumentherapie stabilisiert ist, die Zielparameter der Sepsistherapie nicht erreicht sind und der Patient Vasopressoren benötigt. In diesem Fall ist ein erweitertes Monitoring indiziert (Tab. 2).

Gefordert sind die Überwachung der Vorlast, der Volumenreagibilität und der Gewebsoxygenierung. Die kontinuierliche arterielle Blutdruckmessung ist bei Einsatz von Katecholaminen unbedingt indiziert. Bei diesen Messungen ist zu 
Tab. 2. Monitoring in der Sepsis

\begin{tabular}{|c|c|c|}
\hline \multirow[t]{2}{*}{ Basismonitoring } & \multicolumn{2}{|l|}{ Erweitertes Monitoring } \\
\hline & Vorlast/Volumenreagibilität & Gewebsoxygenierung \\
\hline Arterieller Blutdruck & ZVD (?) & $\mathrm{HZV}$ \\
\hline Herzfrequenz & GEDVI & \\
\hline Diurese & SVV & Blutgasanalyse arteriell \\
\hline Temperatur & EVLW & \\
\hline $\begin{array}{l}\text { Zentralvenöse Sauerstoffsättigung } \\
\text { diskontinuierlich }\end{array}$ & Leg-raising-Test & $\begin{array}{l}\text { zentralvenöse Sauerstoffsättigung } \\
\text { kontinuierlich }\end{array}$ \\
\hline \multirow[t]{3}{*}{ Serum-Laktat } & & Serum-Laktat \\
\hline & $\begin{array}{l}\text { bei klinischem Verdacht auf } \\
\text { abdominelles Kompartmentsyndrom }\end{array}$ & $\begin{array}{l}\text { bei klinischem Verdacht auf } \\
\text { abdominelles Kompartmentsyndrom }\end{array}$ \\
\hline & intraabdomineller Druck & intraabdomineller Druck \\
\hline
\end{tabular}

ZVD = Zentraler Venendruck; GEDVI = globaler Enddiastol-Volumenindex; SVV = Schlagvolumenvarianz; EVLW = extravaskuläres Lungenwasser; HZV = Herzzeitvolumen. beachten, dass sowohl Blutdruck als auch Vorlastparameter von vielen Faktoren beeinflusst sind: unter anderem vom Blutvolumen, vom Gefäßwiderstand, vom intrathorakalen Druck und von Ventrikelfunktion und -Compliance [15].

Der zentrale Venendruck ist als Parameter der Vorlast und Abschätzung der Volumenreagibilität eher nicht geeignet. Massive Anstiege des zentralen Venendrucks unter Volumengabe können ein Hinweis darauf sein, dass eine weitere Volumengabe nicht gut toleriert wird.

Zur Überwachung von Vorlast und Volumenreagibilität sowie des Herzzeit-/Schlagvolumens stehen wenig invasive Monitoring-Systeme zur Verfügung, z.B. das PICCO ${ }^{\circledR}$-System der Firma Pulsion (PULSION Medical Systems mbH, München, Deutschland), aber auch FloTrac ${ }^{\circledR}$-Sensor und Vigileo ${ }^{\circledR}$ System/AV1000 ${ }^{\circledR}$ der Firma Edwards (Edwards Lifescience, Irvine, CA, USA). Beide Systeme ermöglichen die kontinuierliche Messung der zentralvenösen Sauerstoffsättigung sowie des Herzzeit-/Schlagvolumens über die Pulskonturanalyse. Auf die Unterschiede der Systeme soll hier nicht eingegangen werden; hierfür sei auf weiterführende Literatur bzw. auf Lehrbücher der Intensivmedizin verwiesen.

Auch die transösophageale Echokardiographie kann zur Messung des Herzzeitvolumens beim gut sedierten Patienten eingesetzt werden. Bei viszeralchirurgischen Patienten auf der Intensivstation stößt die Methodik an Grenzen: Lageveränderungen des Patienten und liegende Magensonden stören das Messverfahren, und nach Eingriffen am Ösophagus, nach Gastrektomien und bei Ösophagusvarizen ist die Anwendung aus unserer Sicht kontraindiziert.

Das globale enddiastolische Volumen (GEDV) bildet das Blutvolumen aller 4 Herzkammern und somit die Vorlast des gesamten Herzens ab. Verwertbar als Vorlastparameter ist das GEDV vor allem bei Patienten mit normaler Herzfunktion und globalen kardialen Funktionsstörungen. Es korreliert eng mit dem für die diastolische Füllung des Herzens zur Verfügung stehenden zentralen Blutvolumen. Die Verwen- dung von GEDV als Parameter der kardialen Vorlast führte in klinischen Studien zu einer Verkürzung des Aufenthalts auf der Intensivstation; weiterhin konnte ein reduzierter Katecholaminbedarf erreicht werden [16]. Bei isolierter Rechtsherzinsuffizienz ist die Interpretation schwierig (Normwert $680-800 \mathrm{ml} / \mathrm{m}^{2}$ ).

Das extravaskuläre Lungenwasser (EVLW) (Norm 3-7 ml/ $\mathrm{kg} \mathrm{KG}$ ) zeigt einen Anstieg des hydrostatischen Drucks in den Lungen an und steigt bei Zunahme der Kapillarlecks in den Lungen. GEDV und EVLW scheinen zusammen mit anderen Parametern, z.B. Schlagvolumenvarianz (SVV), dazu geeignet, die Volumenzufuhr beim septischen Patienten zu steuern.

Die SVV (Norm unter 10\%) gibt an, um wie viel Prozent das Schlagvolumen über einen bestimmten Messzeitraum hinweg variiert. Die SVV hängt beim beatmeten Patienten im Wesentlichen vom intravasalen Volumenstatus ab. Der Mechanismus beruht darauf, dass es bei Inspiration zu einer intrathorakalen Druckerhöhung und somit zu einer temporären Reduktion der Vorlast kommt. Bei der Expiration nimmt der venöse Rückfluss zum Herzen durch die Druckerniedrigung im Thorax wieder zu; somit steigt auch die Vorlast wieder an. Das heißt, je größer die Varianz, desto größer der Volumenbedarf. Bei Herzrhythmusstörungen ist dieser Parameter nicht zu verwerten, sodass auf andere Vorlastparameter zurückgegriffen werden muss. Die neueste Software erlaubt inzwischen auch die Verwertung der Daten bei Arrhythmien [17]. Messartefakte, z.B. bei einer gedämpften arteriellen Druckkurve, d.h. somit nicht validen Rohdaten, sind zu beachten.

Ein Volumenbelastungstest in Form des passiven Leg-raising-Tests beinhaltet die Gabe von Volumen und die Messung eines Parameters, der den Volumeneffekt anzeigt, z.B. Blutdruck, Schlagvolumen oder Herzzeitvolumen, Herzfrequenz oder Diurese. Der Volumenbelastungstest kann dabei mittels Infusion einer bestimmten Volumenmenge oder durch passives Anheben der Beine des liegenden Patienten erfolgen. Ty- 
Tab. 3. Mögliche Anzeichen einer erfolgreichen und ausreichenden Volumenzufuhr in der Sepsis

\begin{tabular}{ll}
\hline Parameter & Messwert \\
\hline SVV & $<10 \%$ \\
PPV & $<10 \%$ \\
EVLW & steigt, erhöhte Werte \\
GEDVI & steigt, erhöhte Werte \\
HZV & fällt unter Volumenbelastung \\
SV & fällt unter Volumenbelastung \\
ZVD & steigt massiv unter Volumengabe \\
Urinproduktion & $0,5-1$ ml/kg KG/h \\
Serum-Laktat & fällt, normalisiert sich \\
Blutdruck & normalisiert sich \\
\hline
\end{tabular}

SVV = Schlagvolumenvarianz; PPV = Pulsdruckvariation;

EVLW = extravaskuläres Lungenwasser; GEDVI = globaler

Enddiastol-Volumenindex; HZV = Herzzeitvolumen;

$\mathrm{SV}=$ Schlagvolumen; $\mathrm{ZVD}=$ zentraler Venendruck.

pischerweise sind Blutdruck und Schlagvolumen (oder Herzzeitvolumen) die verwandten Surrogatparameter des Leg-raising-Tests, wobei Schlagvolumen und Herzzeitvolumen invasiv oder nichtinvasiv, mittels Pulskonturanalyse und/oder Temperaturverdünnungsmethode gemessen werden können. Wenn das passive Leg-raising-Manöver innerhalb von einer Minute einen Anstieg des Cardiac-Index um mehr als 10\% zeigt, ist eindeutig eine Volumenreagibilität gegeben. Der Leg-raising-Test ist dann positiv. Der Vorteil des Leg-raisingTests ist, dass seine Aussagekraft nicht durch Herzrhythmusstörungen beeinträchtigt wird [14]. Eine Meta-Analyse [18] konnte zeigen, dass die Aussagekraft des passiven Leg-raising-Tests nicht abhängig von der Methode ist, mit der Schlagvolumen oder Herzzeitvolumen gemessen wurden (Pulmonaliskatheter, FloTrac, PICCO, Echokardiographie oder Dopplerultraschall).

Kontinuierliche Messmethoden und wenig invasive Methoden sind für die Messung von Schlagvolumen und Herzzeitvolumen zu bevorzugen, um das Risiko für den Patienten zu minimieren und um schnell auf Veränderungen reagieren zu können.

Dies gilt im Prinzip auch für die Messung der Parameter, die direkt (zentralvenöse Sauerstoffsättigung) oder indirekt (Serum-Laktat) mit der Gewebsoxygenierung assoziiert sind, wobei wir Letzteres bislang ja nur diskontinuierlich messen können.

Die zentralvenöse Sauerstoffsättigung kann als Surrogatparameter der gemischtvenösen Sauerstoffsättigung gelten und ist abhängig vom Sauerstoffangebot und -transport. Werte unter 70\% erfordern die Optimierung durch Volumen- und Katecholamintherapie sowie gegebenenfalls auch eine Verbesserung der Oxygenierung des Patienten.

In Tabelle 3 sind die Anzeichen einer erfolgreichen und ausreichenden Flüssigkeitszufuhr aufgelistet.

\section{Fazit und Konsequenzen}

Ein «Kochrezept» im Sinne von zu erreichenden Normalwerten unter Volumentherapie, die für alle Patienten und Situationen gültig sind, kann für die oben beschriebenen Parameter nicht gegeben werden. Die Frank-Starling-Kurve ist von Vorerkrankungen, Herzinsuffizienz und Katecholaminen beeinflusst. Volumenreagibilität und optimale Vorlast hängen zudem von der aktuellen Erkrankung ab. Die Normwerte aus der Literatur sind somit eher als ungefähre Richtgrößen anzusehen. Trepte et al. [19] stellen fest: «Ein septischer Patient braucht eine andere (höhere) Vorlast als ein Patient nach einem allgemeinchirurgischen elektiven Eingriff und dieser wieder eine andere als ein Patient mit reduzierter kardialer Funktion.» In der Zusammenschau der Messbefunde beim individuellen Patienten, ihrer kritischen Bewertung und der Einbeziehung von Vorerkrankungen sowie der aktuellen Erkrankung liegt wahrscheinlich die Wahrheit.

In einer Meta-Analyse (29 Studien mit 4805 Patienten mit mittleren und großen chirurgischen Eingriffen) zeigten sich unter Verwendung von flussorientierten hämodynamischen Behandlungskonzepten eine Reduktion der postoperativen Komplikationen und eine Tendenz für eine Reduktion der Mortalität [20].

Die Relevanz der Volumentherapie allein für das Therapieergebnis des septischen Patienten lässt sich nicht beziffern. Sicher ist aber, dass eine gesteuerte Therapie, die immer wieder die Volumenreagibilität des Patienten überprüft und die Zielparameter der Sepsistherapie im Auge behält, zu einer Optimierung der Situation des Patienten mit schwerer Sepsis beiträgt. Bei fehlender Volumenreagibilität ist ein gezielter Einsatz positiv inotroper Substanzen nötig, während bei klinischen Zeichen der Überwässerung der Einsatz von Diuretika und gegebenenfalls eine Nierenersatztherapie zu erwägen ist.

\section{Disclosure Statement}

Die Autorin versichert, dass sie keine Industriekooperationen hat, keine Vortragshonorare bekommt oder in den letzten 5 Jahren bekommen hat und keine wissenschaftlichen oder sonstigen Kooperationen mit der Industrie bestehen. 
1 Rivers E, Nguyen B, Havstad S, et al.: Early-goaldirected therapy in the treatment of severe sepsis and septic shock. N Engl J Med 2001;345: 1368-1377.

2 Khoo CK, Vickery CJ, Forsyth N, Vinall NS, EyreBrook IA: A prospective randomized controlled trial of multimodal perioperative management protocol in patients undergoing elective colorectal resection for cancer. Ann Surg 2007;245:867-872.

-3 Brandstrup B, Tønnesen H, Beier-Holgersen R, Hjorts $\emptyset$ E, Ørding H, Lindorff-Larsen K, Rasmussen MS, Lanng C, Wallin L; Danish Study Group on Perioperative Fluid Therapy: Effects of intravenous fluid restriction on postoperative complications: comparison of two perioperative fluid regimens. Ann Surg 2003;238:641-648.

4 Raue W, Haase O, Langelotz C, Neuss H, Müller JM, Schwenk W: Influence of pre-operative fluid infusion on volume status during oesophageal resection - a prospective trial. Acta Anaesthesiol Scand 2008;52:1218-1225.

5 Reinhart K, Brunkhorst FM, Bone HG, et al.: Prevention, diagnosis, therapy and follow-up care of sepsis: 1st revision of S2k guidelines of the German Sepsis Society (Deutsche Sepsis Gesellschaft e.V. (DSG)) and the German Interdisciplinary Association of Intensive Care and Emergency Medicine (Deutsche Interdisziplinäre Vereinigung für Intensiv- und Notfallmedizin (DIVI)). Ger Med Sci 2010;8:Doc14.
6 Hagel S, Brunkhorst F: Sepsis. Intensivmed Notfallmed 2011;48:57-73.

7 Brunkhorst FM, Engel C, Bloos F, et al.: Intensive insulin therapy and pentastarch resuscitation in severe sepsis. N Engl J Med 2008;358:125-139.

8 Perner A, Hasse N, Guttormsen AB, et al.: Hydroxyethyl starch 130/0.42 versus Ringer's acetate in severe sepsis. N Engl J Med 2012;367:124-134.

$\checkmark$ SAFE Study Investigators; Finfer S, McEvoy S, Bellomo R, McArthur C, Myburgh J, Norton R: Impact of albumin compared to saline on organ function and mortality of patients with severe sepsis. Intensive Care Med 2011;37:86-96.

10 Roberts I, Blackhall K, Alderson P, Bunn F, Schierhout G: Human albumin solution for resuscitation and volume expansion in critically ill patients. Cochrane Database Syst Rev 2011;11: CD001208.

11 Wiedermann CJ: Volumentherapeutische Möglichkeiten bei kritisch kranken Patienten. Intensivmed Notfallmed 2011;48:555-568.

12 Heinze H, Hackmann F: Perioperatives Flüssigkeitsmanagement bei Patienten mit großen viszeralchirurgischen Eingriffen. Viszeralmedizin 2011; 27:58-64.

13 Roberts JA, Lipman J: Pharmacokinetic issues for antibiotics in the critically ill patient. Crit Care Med 2009;37:840-851.

14 Empfehlungen der World Society of the Abdominal Compartment Syndrome. www.wsacs.org.
5 Shujaat A, Bajwa AA: Optimization of preload in severe sepsis and septic shock. Crit Care Res Pract 2012;2012:761051.

16 Goepfert MS, Reuter DA, Akyol D, Lamm P, Kilger E, Goetz AE: Goal-directed fluid management reduces vasopressor and catecholamine use in cardiac surgery patients. Intensive Care Med 2007:33:96-103.

17 Cannesson M, Tran NP, Cho M, Hatih F, Michard F: Predicting fluid responsiveness with stroke volume variation despite multiple extrasystoles. Crit Care Med 2012;40:193-198.

18 Cavallaro F, Sandroni C, Marano C, et al.: Diagnostic accuracy of passive leg raising for prediction of fluid responsiveness in adults: systematic review and meta-analysis of clinical studies. Intensive Care Med 2010;36:1475-1483.

19 Trepte CJC, Haas SA, Reuter DA: Steuerung der Volumentherapie - Was wird die Rivers-Kriterien ersetzen?; in Markewitz A, Muhl E, Putensen C, Quintel M, Sybrecht GW (Hrsg): DIVI-Jahrbuch 2012/2013. Berlin, Medizinisch Wissenschaftliche Verlagsgesellschaft, 2013, pp 61-67.

20 Hamilton MA, Cecconi M, Rhodes A: A systematic review and meta-analysis on the use of preemptive hemodynamic intervention to improve postoperative outcomes in high-risk surgical patients. Anesth Analg 2011;112:1392-1402. 